The Psychological Record, 2011, 61, 287-312

\title{
ESTABLISHING CONTEXTUAL CONTROL OVER SYMMETRY AND ASYMMETRY PERFORMANCES IN TYPICALLY DEVELOPING CHILDREN AND CHILDREN WITH AUTISM
}

\author{
Jennifer O'Connor \\ Applied Behavior Analysis Centre for Autism Schooling (ABACAS), Kilbarrack \\ Yvonne Barnes-Holmes and Dermot Barnes-Holmes \\ National University of Ireland, Maynooth
}

\begin{abstract}
Experiments 1, 2, and 3 investigated generalized contextually controlled symmetry and asymmetry in typically developing children and children with autism. In Experiment 1, eight typically developing children demonstrated the target performances without intervention. In Experiment 2, multiple-exemplar training and the use of familiar stimuli appeared to be effective in establishing the target performances for some of the children with autism. Experiment 3 examined an alternative intervention, based on alternating between correct and incorrect responding, for children with autism who appeared to have even greater difficulties in establishing contextually controlled symmetry and asymmetry. The findings are discussed in the context of the existing literature and interventions for establishing new repertoires in children with developmental disabilities.
\end{abstract}

Key words: conditional discriminations, contextual control, autism

Numerous studies have examined the integrity versus instability of equivalence and its component relations, and the systematic reversal of baseline training is a common methodology for such investigations (Sidman, 1986; Wulfert \& Hayes, 1988). Outcomes associated with these manipulations have varied. Specifically, in some studies symmetry relations changed in accordance with the new conditional discriminations, while equivalence remained intact (Pilgrim \& Galizio, 1990; Saunders, Saunders, Kirby, \& Spradlin, 1988). In other studies, conditional discriminations became inconsistent (Pilgrim, Chambers, \& Galizio, 1995). In attempting to account for the various findings, researchers have attributed experimental influence to a range of sources, including procedural differences, demand characteristics, stimulus naming, and participant age.

Carrigan and Sidman (1992) accounted for the different outcomes from reversal training by appealing to a distinction between Type $S$ and Type $R$

Correspondence concerning this article should be addressed to Yvonne Barnes-Holmes, Department of Psychology, National University of Ireland, Maynooth, Maynooth, Co. Kildare, Ireland.E-mail: Yvonne.barnes-holmes@nuim.ie 
responding. In short, they argued that conditional discrimination procedures generate conditional relations between samples and negative comparison stimuli, as well as between samples and positive comparison stimuli. They referred to the relation between sample and positive comparison stimuli as the select or Type $S$ relation and the relation between sample and incorrect comparison stimuli as a reject or Type $R$ relation. According to this view, a conditional discrimination may reflect one pattern or the other because the procedure neither restricts nor encourages either one. Although Carrigan and Sidman's analysis predicted different results for reflexivity and transitivity when under Type S or Type R control, they hypothesized that both patterns yield the same outcomes in symmetry tests. For example, train A1-B1-C1 and $\mathrm{A} 2-\mathrm{B} 2-\mathrm{C} 2$ and then reverse the contingencies to train A1-B2-C1 and A2-B1$\mathrm{C} 2$. The symmetry responses in the first stage would be B1-A1 and B2-A2 but in the second stage would be B2-A1 and B1-A2. However, in both stages equivalence would involve $\mathrm{C} 1-\mathrm{A} 1$ and $\mathrm{C} 2-\mathrm{A} 2$ responding. As such, Carrigan and Sidman argued that a contingency reversal in one of the conditional discriminations changes control in that discrimination from Type $\mathrm{S}$ to Type $\mathrm{R}$, thus reversing symmetry performances but leaving equivalence unchanged. As a result, symmetry probes provide no indication of which pattern controls responding (see also Johnson \& Sidman, 1993).

Using the Wisconsin General Test Apparatus (WGTA) with undergraduate students, Pilgrim and Galizio (1995) established two four-member classes, followed by reversal of the A-D relations. This resulted in changes to symmetry but left transitivity and equivalence intact. These findings paralleled those from the 1990 study by the same researchers. However, the researchers argued that their findings did not support the view that stimulus control had shifted completely from Type S to Type R at any point, but they would not rule out a complex mix of both types of control.

A number of studies have also examined the extent to which equivalence responding and its component relations are more or less malleable in children than adults. For example, Pilgrim et al. (1995) used the WGTA with typically developing children ages 5 to 7 years old. The results demonstrated that the establishment of the new discriminations after the reversal was less effective when presented within a full-probe baseline arrangement, even though this had been sufficient for the adults in the previous study. That is, the adults' symmetry performances had been tightly controlled by contingencies of the baseline relations currently in effect, but this pattern was recorded only with the oldest of the eight children. Furthermore, although five children showed the reversed performances, responding was inconsistent and even non-manipulated relations were disrupted. The authors argued that the more consistent pattern of responding recorded with adults was evidence of class modification, whereas the inconsistent performances of the children showed disruption of the original classes and some degree of class reorganization. In short, they argued that the children's equivalence classes were more fragile than malleable.

To some extent, Pilgrim et al.'s (1995) conclusion supported earlier findings by Michael and Bernstein (1991), who reported disrupted equivalence and inconsistencies in conditional discriminations after reversal training with 4- and 5-year-olds. However, Spradlin, Saunders, and Saunders (1992) established five-member classes with 8- to 12-year-olds and found that reversal of one conditional discrimination did not affect equivalence. In accounting for their data, the latter authors suggested that larger classes were less susceptible to disruption by reversing a single relation. 
In a study of adolescents and adults with developmental disabilities, Saunders et al. (1988) reported that reversal training did not affect equivalence for two participants, although some disruption was observed with a third. In a subsequent study, Saunders, Drake, and Spradlin (1999) established five-member classes in children ages 3 to 5 years old and reported that successful equivalence outcomes were more likely to result from comparison-asnode $(\mathrm{CaN})$ than sample-as-node $(\mathrm{SaN})$ training. As a result, they argued that training structure is a factor that affects equivalence formation, at least with larger classes and children. In a second study, there was evidence of class expansion when the children showed reversal of the conditional discriminations, but no disruption to symmetry or equivalence.

In a specific attempt to examine the potential role of naming in equivalence, Carr and Blackman (2001) employed a reversal procedure that involved adding two novel comparison stimuli to opposing members of equivalence classes with undergraduates. Although all early relations were readily established, a variety of alternative response patterns emerged after the reversal training. Specifically, three participants showed minimal disruption to symmetry and transitivity, one showed reversal of symmetry while equivalence remained intact, and the remaining two showed reversal of both symmetry and transitivity. Furthermore, five of the participants demonstrated the maintenance of the original conditional discriminations (these were completely reversed for the remaining participant). In a second study, three participants reversed symmetry and transitivity, three reversed symmetry but transitivity remained intact, and all showed maintenance of the original conditional discriminations. Finally, in a third study, two participants maintained symmetry and transitivity, one reversed symmetry but maintained equivalence and transitivity, one showed disrupted symmetry but maintenance of transitivity, and one reversed symmetry and showed disrupted transitivity.

In summary, several patterns have been recorded after reversals of conditional discriminations with both adults and children. To account for the wide variations in the findings, several hypotheses have proposed possible sources of experimental influence, as well as generic participant variables. At a higher level, the disparity of findings poses questions about the extent to which symmetry and equivalence relations are sensitive to contingencies, thus raising further questions about the extent to which these behaviors may be defined as operant responding.

Barnes-Holmes, Barnes-Holmes, Roche, and Smeets (2001a, 2001b) investigated symmetry in children ages 4 to 5 years old. Across eight experiments, 24 of 30 children failed to demonstrate the target symmetry performances prior to multiple-exemplar training but easily did so afterwards. The researchers argued that the findings were consistent with relational frame theory (RFT) predictions that multiple-exemplar training is effective in facilitating a derived transformation of functions in accordance with symmetry, but also argued that the data support the view of symmetry as a type of operant behavior.

Several other researchers have disagreed with the latter conclusion in particular and have attempted to show that derived symmetry is not operant responding because it shows insensitivity to operant contingencies. For example, Boelens, Van Den Broek, and Calmeyn (2003) investigated symmetry in young children using both unfamiliar (Experiment 1) and familiar (Experiments 2 and 3) stimulus sets. Moreover, the researchers attempted to strengthen or weaken symmetry performances by arranging pretraining contingencies in which either symmetry or asymmetry responding was reinforced, respectively. 
The children were first explicitly trained to name a picture, thus establishing the picture-name relations (P1-N1 and P2-N2). Following this name training, they were exposed to a form of pretraining in either symmetry or asymmetry employing the same stimulus classes. Specifically, the children exposed to the symmetry pretraining were trained to emit the symmetric relations N1-P1 and N2-P2, whereas the children exposed to the asymmetry pretraining were explicitly trained to emit the asymmetric relations N1-P2 and N2-P1. After pretraining, the children were exposed to a visual match-to-sample (MTS) task employing pictures, and all were tested for symmetry relations in this context. The results demonstrated that all children responded symmetrically on the tests, thus showing no apparent impact of the asymmetry pretraining on subsequent symmetry performances. The researchers concluded that this was evidence of the generality of children's symmetry performances, which were not affected by the contingencies operating during the pretraining.

One way to investigate the operant nature of symmetry responding and the variables that potentially control it is to determine whether symmetry performances can be brought under contextual control. There have been a number of studies of complex forms of contextual control over relational responding, including the relating of relations and the relating of relational networks to relational networks (Stewart, Barnes-Holmes, Roche, \& Smeets, 2001). Dougher, Perkins, Greenway, Koons, and Chiasson (2002) reported evidence of derived contextually controlled equivalence in adults. In the first part of the study, the researchers established three three-member classes using unfamiliar stimuli. They then trained a conditional stimulus function in the presence of one member of each class and employed a multiple-exemplar training procedure to establish contextually controlled equivalence with novel stimuli. The results indicated that contextually controlled equivalence relations could be established with unfamiliar stimuli and that, once established, these performances could be generalized to novel equivalence classes. The findings supported the RFT view that equivalence constitutes a type of generalized operant behavior that is subject to contextual control. Similar findings have also been reported by Meehan and Fields (1995). In spite of the importance of contextual control in symmetry, only one study to date has attempted to establish contextual control over symmetry in children.

The current study attempted to establish repertoires of contextually controlled symmetric and asymmetric responding in typically developing children and children diagnosed with autism. In doing so, the work also sought to develop educational interventions that may prove useful in remediating deficits in the target skills, especially with populations diagnosed with developmental disabilities.

\section{Experiment 1}

\section{Participants}

Eight typically developing children (referred to as Participants 1-8) participated in Experiment 1. The three girls and five boys were between 6 and 9 years old. All were selected on the basis that neither their parents nor their teachers had identified them as presenting with a learning difficulty. All children were from the same mainstream primary school in Dublin, Ireland. 


\section{Setting}

All of the experimental trials in which the children participated were conducted in a small classroom within the school. All participation was on an individual basis, and only the experimenter and the participant were present in the room. During all trials, the experimenter was seated beside the child at a small experimental table.

\section{Materials}

Experiment 1 involved the use of two stimulus sets, each of which comprised two classes (see Table 1). The sets differed in terms of the actual stimuli they contained and the point in the experimental sequence at which they were presented (Set 1 was always presented first). In Set 1, both of the A stimuli (A1 and A2) were written nonsense syllables printed in black ink on a white card, and the B stimuli (B1 and B2) were their spoken counterparts (e.g., VUG and "vug," respectively).

\begin{tabular}{|c|c|c|c|c|}
\hline \multirow{2}{*}{ Set 1} & A1 & VUG & $A 2$ & LUP \\
\hline & B1 & "vug" & B2 & "lup" \\
\hline \multirow{2}{*}{ Set 2} & A3 & & A4 & \\
\hline & B3 & & B4 & \pm \\
\hline \multirow{2}{*}{ Set 3} & A5 & RUP & $A 6$ & TER \\
\hline & B5 & "rup" & B6 & "ter" \\
\hline \multirow{2}{*}{ Set 4} & A7 & & A8 & $\leadsto$ \\
\hline & B7 & & B8 & $G$ \\
\hline \multirow{2}{*}{ Set 5} & A9 & QEG & A10 & $\mathrm{SOZ}$ \\
\hline & B9 & "qeg" & B10 & "SOz" \\
\hline \multirow{2}{*}{ Set 6} & A11 & $\checkmark$ & $\mathrm{A} 12$ & $\varnothing$ \\
\hline & B11 & б & $\mathrm{B} 12$ & $\mu$ \\
\hline \multirow{2}{*}{ Set 7} & A13 & HORSE & A14 & CHICKEN \\
\hline & B13 & "horse" & B14 & "chicken" \\
\hline \multirow{2}{*}{ Set 8} & A15 & BED & A16 & CAT \\
\hline & B15 & "bed" & B16 & "cat" \\
\hline \multirow{2}{*}{ Set 9} & A17 & BOY & A18 & GREEN \\
\hline & $\mathrm{B} 17$ & "boy" & $\mathrm{B} 18$ & "green" \\
\hline \multirow{2}{*}{ Set 10} & A19 & APPLE & A20 & TRACTOR \\
\hline & B19 & "apple" & B20 & "tractor" \\
\hline
\end{tabular}

All of the stimuli contained within Set 2 were also depicted on white cards, identical in size to Set 1 , but were black, two-dimensional abstract figures. Unlike the matching A and B stimuli from Set 1, the A and B stimuli here did not relate to one another in any formal way.

Two stimuli were also employed in Experiment 1 as contextual cues (i.e., $\mathrm{C} 1$ and $\mathrm{C} 2$ ) for the control of the children's symmetrical and asymmetrical 
responses. The cues were identical in form, and both were cardboard circles that were $25 \mathrm{~cm}$ in diameter with a small ridge glued to the back to enable the card to stand independently. They differed only in color (one was red and the other was blue). A range of small edible items, including sweets and crisps, were used as reinforcers for correct responding.

\section{Experimental Sequence}

The experimental sequence contained Phases 1 through 5, presented in that order (see Table 2). Phase 1 involved explicit training of the appropriate textual responses to the two printed nonsense syllables (name training) employed as A stimuli in Set 1 (A1-B1 and A2-B2). In Phase 2, participants received explicit training of both the symmetrical (B1-A1, $\mathrm{B} 2-\mathrm{A} 2)$ and asymmetrical (B1-A2, B2-A1) relations, using the colored circles as contextual cues. For example, in the presence of the blue circle (C1), reinforcement was provided for responding in accordance with the symmetrical relations, but in the presence of the red circle (C2), reinforcement was provided for responding in accordance with the asymmetrical relations.

Table 2

The Experimental Sequence Employed in Experiment 1

\begin{tabular}{ccc}
\hline Phase 1 & Explicit name training (written-spoken) & A1-B1 \& A2-B2 \\
Phase 2 & Train symmetry (C1) and asymmetry (C2) & C1 (B1-A1 \& B2-A2) \\
& under contextual control & C2 (B1-A2 \& B2-A1) \\
Phase 3 & Test symmetry (C1) and asymmetry (C2) & C1 (B1-A1 \& B2-A2) \\
Phase 4 & under contextual control & C2 (B1-A2 \& B2-A1) \\
& $\begin{array}{c}\text { Conditional discrimination MTS training } \\
\text { Test generalized symmetry (C1) }\end{array}$ & A3-B3 \& A4-B4 \\
Phase 5 & and asymmetry (C2) under contextual & C1 (B3-A3 \& B4-A4) \\
& control in MTS & C2 (B3-A4 \& B4-A3) \\
\hline
\end{tabular}

Phase 3 then consisted of a test of the previously trained B-A symmetrical and asymmetrical relations under contextual control. Phases 4 and 5 examined the generalization and contextual control of these performances using a novel set of stimuli. Specifically, in Phase 4, participants were exposed to MTS training trials involving the abstract stimuli from Set 2 in order to establish the conditional discriminations A3-B3 and A4B4 (see Table 1). In Phase 5, they were then exposed to a test of responding in accordance with the contextually controlled symmetrical (B3-A3 and B4-A4) and asymmetrical (B3-A4 and B4-A3) relations. All eight children completed the study in only one experimental session that lasted approximately $20 \mathrm{~min}$, and at no point did any child indicate a wish to terminate participation.

\section{Corrective Feedback}

Corrective feedback was provided after all training trials but after none of the test trials. Corrective feedback for correct responses consisted of verbal praise (e.g., "good girl/boy," "well done," "great”). Corrective feedback for incorrect responses involved the experimenter simply repeating the antecedent (e.g., repeating the words "goes with" in Phase 4) and 
modeling the correct response (e.g., by touching the correct comparison stimulus). During trials in which corrective feedback was provided for incorrect responding, participants were not asked to repeat the trial. After corrective feedback and modeling as appropriate, the next trial was presented immediately.

\section{Procedure}

Phase 1: Explicit name training. Participants were first trained explicitly to emit textual responses to the written nonsense syllables from Set 1 (A1: VUG, A2: LUP). Training consisted of 20 trials, 10 for each A stimulus. During the first training trial for each syllable, the printed card (e.g., A1) was held up in front of the child and the experimenter emitted the appropriate textual response (e.g., "vug"). In order to respond correctly, the child was required to repeat the echoic response (e.g., "vug") within $10 \mathrm{~s}$ of the instruction. For the remaining 18 trials, the experimenter did not emit the target textual response; hence the child was required to emit a textual response rather than an echoic response.

Corrective feedback followed each training trial in Phase 1, after which the card was removed and the next trial was presented immediately. Each of the two cards was randomly presented 10 times across a block of 20 trials. Participants were required to produce a minimum of 8 out of 10 correct responses for each A stimulus before proceeding to Phase 2.

Phase 2: Training symmetry and asymmetry under contextual control. In Phase 2, participants were exposed to explicit training of the contextually controlled symmetrical and asymmetrical relations involving the nonsense syllables from Phase 1 . That is, the symmetrical relations $\mathrm{B} 1-\mathrm{A} 1$ and $\mathrm{B} 2-\mathrm{A} 2$ and the asymmetrical relations $\mathrm{B} 1-\mathrm{A} 2$ and $\mathrm{B} 2-\mathrm{A} 1$ were trained explicitly under the contextual control of $\mathrm{C} 1$ and $\mathrm{C} 2$. At the beginning of Phase 2, participants were given the following instructions:

I am going to place these two words [experimenter places the cards down containing the written A stimuli] on the table in front of you and I'll say a name. Then you have to choose the card that you think is the right one. If you get it right, I will tell you, and I can also tell you if you get it wrong.

The two cards containing the A stimuli were placed beside each other on the table, with their locations randomized across trials. The specific circle designated for the control of either symmetrical or asymmetrical relations was also counterbalanced across participants.

Immediately after the two cards were placed on the table, the experimenter placed one of the contextual cues directly above and between the cards. The experimenter then emitted a vocal name that was either B1 or B2 ("vug" or "lup"), and participants were required to point to or touch the appropriate written nonsense syllable in the presence of that cue. Thus, when blue was designated for the control of symmetry (and red for asymmetry), and the experimenter said "vug" in the presence of the blue cue, a correct response involved selecting the VUG card (symmetry). In the presence of a red cue, a correct response involved selecting the LUP card (asymmetry). Phase 2 consisted of 40 trials, 20 for training symmetry (10 each of B1-A1 and B2-A2) and 20 for asymmetry (10 each of B1-A2 and B2-A1). All trials were 
identical in format, except that the contingencies were reversed between symmetry and asymmetry. Participants were required to demonstrate a mastery criterion of 36 out of 40 correct, with no more than one error on each of the two types of symmetry or asymmetry trials. Children who reached this criterion proceeded immediately to Phase 3. Those who did not were reexposed immediately to Phase 1 , with a view to then receiving a second exposure to Phase 2.

Phase 3: Testing symmetry and asymmetry under contextual control. Phase 3 was designed to test the contextual control of the symmetrical and asymmetrical response patterns that had been explicitly trained in Phase 2 . Phase 3 was identical in format to Phase 2, except that there were test (rather than training) trials and no feedback. The test comprised a total of 20 trials, with five exposures to each of the four trial types (B1-A1, B2-A2 and B1-A2, B2-A1). Participants received the following instructions:

This time, I am going to place the two words in front of you as I did before and then I'll say a name. Once again you have to choose the card that you think is the right one. The only difference is that this time, I can't tell you if you got it right or wrong.

In order to pass the test, participants were required to produce 16 out of 20 correct responses, with no more than one error on each trial type. Children who passed proceeded immediately to Phase 4 . Those who failed were returned to Phase 2.

Phase 4: MTS training. In Phases 4 and 5, the children were exposed to a novel tabletop MTS task that examined the generalization and contextual control of the target symmetry and asymmetry performances on a novel set of stimuli (Set 2). During Phase 4, novel A-B relations (A3-B3 and A4-B4) were explicitly trained. Prior to the first trial, participants received the following instructions:

Now we are going to do another task, and this is about matching things together. Like the task we did earlier, I will be able to tell you when you get them right or wrong.

Phase 4 consisted of 20 randomized MTS training trials, 10 for A3-B3 and 10 for A4-B4. During each trial, the experimenter first placed the sample card down on the table, followed by the two comparison stimuli below it, one on the left and the other on the right (locations counterbalanced across trials). The experimenter then touched the sample (e.g., A3) and immediately presented the antecedent "goes with." The experimenter then remained looking at the sample for $10 \mathrm{~s}$, during which the child was required to make a selection response from one of the comparison stimuli. During this time, the experimenter did not look at the child or otherwise interact in any way. A correct response involved the child indicating the correct comparison, either by pointing to it or picking it up. Corrective feedback was presented after each trial. As in previous phases, the mastery criterion was 18 out of 20 correct responses, with no more than one error on each trial type, after which participants proceeded immediately to Phase 5. It was anticipated that the training would continue until all children had reached criterion and the two classes had been established.

Phase 5: Contextually controlled MTS testing. Phase 5 was identical in format to the previous phase, except that the contextual cues were 
reemployed. Symmetrical responding involved participants selecting B3 in the presence of A3 and B4 in the presence of A4 in conjunction with the appropriate contextual cue. Which cue controlled which type of responding depended exactly on the training from the initial phases in order to ensure consistency in the contextual control. Conversely, asymmetrical responding involved selecting B3 in the presence of A4 and B4 in the presence of A3 in conjunction with the appropriate contextual cue.

Phase 5 consisted of 20 test trials, 10 for contextually controlled symmetry (five B3-A3 and five B4-A4) and 10 for asymmetry (five B3-A4 and five B4-A3). As in Phase 3, no test trials were consequated with corrective feedback. At the beginning of Phase 5, the children were instructed as follows:

This time, we are going to do the matching task as we did before, but the only difference is that I can't tell you if you got it right or wrong.

Passing the test required 16 out of 20 correct responses, with no more than one error on each trial type. Children who passed the test at this point had completed their participation in the study. Children who failed were returned to Phase 1.

\section{Interobserver Agreement}

Twenty-five percent of all test trials and 30\% of all training trials were recorded by an independent observer who was located at the opposite end of the experimental table from the participant and experimenter. This observer did not interact with the children in any way during the sessions. There was $100 \%$ agreement on all trials observed by both the experimenter and the independent observer.

\section{Results and Discussion}

The total number of training trials required to reach criterion and the total number of correct test trials for each participant across all five phases in Experiment 1 are presented in Table 3.

Table 3

The Training and Test Performances Recorded With Participants 1-8 Across All Phases in Experiment 1

\begin{tabular}{cccccc}
\hline Participant & $\begin{array}{c}\text { Phase 1 } \\
\text { training (20) }\end{array}$ & $\begin{array}{c}\text { Phase 2 } \\
\text { training (40) }\end{array}$ & $\begin{array}{c}\text { Phase 3 } \\
\text { testing (20) }\end{array}$ & $\begin{array}{c}\text { Phase 4 } \\
\text { training (20) }\end{array}$ & $\begin{array}{c}\text { Phase 5 } \\
\text { testing (20) }\end{array}$ \\
\hline 1 & 20 & 38 & 20 & 20 & 20 \\
2 & 20 & 36 & 19 & 20 & 19 \\
3 & 20 & 36 & 20 & 20 & 20 \\
4 & 20 & 38 & 18 & 20 & 20 \\
5 & 20 & 39 & 20 & 20 & 19 \\
6 & 20 & 36 & 20 & 20 & 20 \\
7 & 20 & 36 & 20 & 20 & 20 \\
8 & 20 & 37 & 20 & 19 & 19 \\
\hline
\end{tabular}

Prior to Phase 1, all eight typically developing children produced perfect echoic responses in the presence of the printed words A1 and A2 (not 
shown). They then produced high levels of accuracy in all five phases, with no reexposures to any phase. Put simply, all eight children readily learned and generalized the target contextually controlled symmetrical and asymmetrical performances.

Of course, the exemplary performances of this sample of children is hardly surprising given that they were all between 6 and 9 years old and were all typically developing. In contrast, Experiment 2 involved conducting the same experiment with a group of children with autism. In the case of the latter, we were largely concerned with identifying the types of intervention that might be employed when training was not immediately successful or when the test performances did not emerge.

\section{Experiment 2}

\section{Participants}

Ten children (nine boys and one girl), ages 6 to 9 years, referred to as Participants 9 through 18, participated in Experiment 2. All attended CABAS (Comprehensive Application of Behavior Analysis to Schooling) educational facilities in Dublin, Drogheda, or Cork in Ireland and had been independently diagnosed as showing a mild to moderate level of autism. The children were classified in the CABAS facilities according to their individual levels of verbal ability (see Greer \& Ross, 2007, for a comprehensive review), and all of the participants were categorized as reader/writers with emergent levels of self-editing skills. In simple terms, these children had relatively high levels of verbal ability.

\section{Setting}

The setting employed in Experiment 2 was identical to the setting in Experiment 1.

\section{Materials}

All 10 children who participated in Experiment 2 were exposed to Sets 1 and 2 from the previous experiment. However, in order to accommodate difficulties presented by several children, eight additional stimulus sets were employed (see Table 1). Sets 3 and 5 were similar to Set 1 and involved matched written and spoken nonsense syllables. Sets 4 and 6 were similar to Set 2, and each was comprised of four unmatched abstract shapes. Experiment 2 also involved the use of four additional stimulus sets (Sets 7-10) that were similar in construction to Set 1 but contained matched written and spoken words (e.g., HORSE and "horse"), rather than nonsense syllables.

All 10 participants involved in Experiment 2 were engaged in an individual token economy as part of their CABAS education. The tokens employed were small plastic disks of various colors that were used as reinforcers for all responses. Each participant was also required to fill a token mat (a mat with designated spaces for inserting tokens when earned) before exchanging tokens for reinforcers. 


\section{Experimental Sequence}

The experimental sequence was identical to Experiment 1, except that the children required at least two, and in some cases more than 30, separate sessions in order to complete their participation. Each session lasted no more than $20 \mathrm{~min}$. If at any point during training a child indicated the desire to stop, the experimental trials were terminated immediately for that session, and those trials were repeated in the following session.

\section{Corrective Feedback}

The corrective feedback was identical to Experiment 1, except that correct responses were also accompanied by the presentation of tokens.

\section{Procedure}

The basic procedure was identical to Experiment 1, except that two key interventions were necessary to establish the target performances for some children. These included multiple-exemplar training as a sixth training phase and the use of familiar stimuli.

Phase 6: Contextually controlled MTS training. Participants who failed to pass the generalization test on their first exposure to Phase 5 were systematically exposed to an additional training phase of the target performances. This simply involved using the same abstract stimuli from Set 2 but now consequating each trial with corrective feedback and access to tokens. When criterion was reached $(18 / 20)$, participants were then reexposed to all five phases with novel stimulus sets (Sets 3 and 4) to determine the efficacy of the exemplar training in Phase 6. It was intended that this pattern of explicit training and then testing on a new set would continue until each participant had produced a successful test performance in Phase 5 with a novel stimulus set (hence the use of the term exemplar training).

For some children, exemplar training was introduced according to a type of multiple-baseline design across participants. That is, the first child to fail the Phase 5 test received exemplar training immediately, whereas subsequent children who failed were exposed to exemplar training only after repeated exposures to Phase 5. This design enabled us to determine whether or not the target performances would emerge as a result of repeated exposures to Phase 5 alone, prior to the introduction of Phase 6, or whether the exemplar training was necessary for these performances to be established. With a number of children, such a design was not employed because they had already received a great number of exposures to earlier phases.

\section{Interobserver Agreement}

Twenty-five percent of all test trials and 30\% of all training trials in Experiment 2 were recorded by an independent observer located at the opposite end of the table from the participant and experimenter. This observer did not interact with the children in any way during the sessions. There was $100 \%$ agreement on all trials. 


\section{Results and Discussion}

Four children (Participants 9, 10, 11, and 12) required no interventions; three (Participants 13,14, and 15) required only the multiple-exemplar training in Phase 6; and three (Participants 16, 17, and 18) required both exemplar training and the use of familiar stimuli in order to demonstrate the target performances. The results for each of these three groups are described separately.

Participants 9, 10, 11, and 12. The data recorded with Participants 9, 10, 11, and 12 are presented in Table 4 . These children required between one and two experimental sessions in total and performed in much the same way as the typically developing children in Experiment 1. For example, Participant 11 produced a pattern of responding that was most like the previous children, proceeding rapidly through all five phases in a single exposure.

Table 4

The Training and Test Performances Recorded With Participants 9-12 Across All Phases in Experiment 2

\begin{tabular}{cccccc}
\hline Participant & $\begin{array}{c}\text { Phase 1 } \\
\text { training (20) }\end{array}$ & $\begin{array}{c}\text { Phase 2 } \\
\text { training (40) }\end{array}$ & $\begin{array}{c}\text { Phase 3 } \\
\text { testing (20) }\end{array}$ & $\begin{array}{c}\text { Phase 4 } \\
\text { training (20) }\end{array}$ & $\begin{array}{c}\text { Phase 5 } \\
\text { testing (20) }\end{array}$ \\
\hline 9 & 20 & 33 & - & - & - \\
10 & 20 & 40 & 19 & 19 & 20 \\
\hline 11 & 9 & - & - & - & - \\
\hline 12 & 19 & 36 & 19 & 20 & 18 \\
\hline & 20 & 37 & 18 & 17 & 19 \\
\hline
\end{tabular}

Note. Dashed lines (-) indicate absence of exposure to a particular stimulus set. Each line represents an individual experimental session.

Although the remaining three children in this group showed highly competent performances (none required Phase 6), there were some minor differences among them. For example, Participants 9 and 12 produced perfect training performances in Phase 1 but then failed to reach criterion on the contextual control training. However, they each mastered this after a second exposure to both phases and then proceeded immediately through all subsequent training and testing. Somewhat similarly, Participant 10 failed to reach criterion on his first exposure to Phase 1 , but after a second exposure he proceeded quickly through all subsequent phases. Four of the children with autism, therefore, readily demonstrated generalized derived contextually controlled symmetry and asymmetry responding without explicit intervention.

Participants 13, 14, and 15. Participants 13, 14, and 15 showed greater difficulty than the other children, particularly in the latter phases. The data recorded for these three children is presented in Table 5. 
Table 5

The Training and Test Performances Recorded With Participants 13-15 Across All Phases in Experiment 2

\begin{tabular}{|c|c|c|c|c|c|c|c|}
\hline Participant & Sets & $\begin{array}{l}\text { Phase } 1 \\
\text { training } \\
(20)\end{array}$ & $\begin{array}{c}\text { Phase } 2 \\
\text { training } \\
(40)\end{array}$ & $\begin{array}{l}\text { Phase } 3 \\
\text { testing } \\
(20)\end{array}$ & $\begin{array}{c}\text { Phase } 4 \\
\text { training } \\
(20)\end{array}$ & $\begin{array}{c}\text { Phase } 5 \\
\text { testing } \\
(20)\end{array}$ & $\begin{array}{c}\text { Phase 6 } \\
\text { training } \\
(20)\end{array}$ \\
\hline \multirow{5}{*}{13} & \multirow{3}{*}{$1 \& 2$} & 20 & 27 & - & - & - & - \\
\hline & & 20 & 38 & 20 & 13 & - & - \\
\hline & & 20 & 39 & 20 & 18 & 14 & 18 \\
\hline & $3 \& 4$ & 20 & 39 & 20 & 20 & 17 & 18 \\
\hline & $5 \& 6$ & 20 & 39 & 20 & 19 & 19 & - \\
\hline \multirow{7}{*}{14} & \multirow{6}{*}{$1 \& 2$} & 20 & 23 & - & - & - & - \\
\hline & & 20 & 37 & 18 & 19 & 7 & - \\
\hline & & 20 & 39 & 18 & 19 & 16 & - \\
\hline & & 20 & 37 & 17 & - & - & - \\
\hline & & 20 & 38 & 17 & - & - & - \\
\hline & & 19 & 39 & 19 & 19 & 17 & 20 \\
\hline & $3 \& 4$ & 20 & 40 & 20 & 19 & 20 & - \\
\hline \multirow{6}{*}{15} & \multirow{5}{*}{$1 \& 2$} & 20 & 36 & 20 & 18 & 5 & - \\
\hline & & 20 & 34 & - & - & - & - \\
\hline & & 20 & 38 & 18 & 19 & 0 & - \\
\hline & & 20 & 40 & 20 & 20 & 0 & - \\
\hline & & 20 & 38 & 20 & 19 & 0 & 19 \\
\hline & $3 \& 4$ & 20 & 40 & 20 & 19 & 20 & - \\
\hline
\end{tabular}

Note. Dashed lines (-) indicate absence of exposure to a particular stimulus set. Each line represents an individual experimental session.

For illustrative purposes, consider the performances of Participant 13. He required two exposures to complete the contextual control training in Phase 2 (nonsense syllables) and two exposures to complete the MTS training in Phase 4 (abstract shapes), but then he still failed the generalization test in Phase 5 (14/20). At this point, he was exposed to multiple-exemplar training in Phase 6, using the same stimuli from Phase 5. He reached the training criterion within the minimum number of trials (20) and was then reexposed to all five phases using novel sets (Sets 3 and 4). Unfortunately, he was just one response short of passing the test in his second exposure to Phase 5, so he was reexposed to exemplar training, which he again completed in the minimum number of trials. He was then reexposed to the five phases with further novel stimulus sets (Sets 5 and 6) and passed the test on this occasion.

Participant 14 also needed two exposures to complete the contextual control training in Phase 2 but demonstrated no difficulty with the MTS 
training in Phase 4 . Nonetheless, he significantly failed the first exposure to the generalization test (7/20), and thus, in line with the multiplebaseline design, he received a second exposure to all five phases using the same sets. Although his second performance on the generalization test was considerably improved (16/20), it remained insufficient to pass. Hence, he received a third exposure, but his performance on the contextual control test in Phase 3 fell just short of passing. This performance was repeated on a fourth exposure. On his fifth exposure to Phase 3 , he passed again but narrowly missed criterion on the generalization test. At this point, he received explicit exemplar training (Phase 6) and easily passed the test on a subsequent exposure with novel sets.

Participant 15 reached Phase 5 immediately but produced a very weak test performance (5/20). On his second exposure, his performance weakened in Phase 2, but this was rectified by a third exposure. At this point, however, his test performance in Phase 5 fell to 0/20, and in line with the multiple-baseline design, he was reexposed to all five phases on two more occasions. However, his performances remained at 0/20 throughout. After his fourth exposure to Phase 5, he received exemplar training and produced excellent performances throughout on novel sets.

In summary, Participants 13, 14, and 15 showed greater difficulty overall than all previous participants. Although their occasional weaknesses in the early phases were easily rectified by repeated exposures, this was not the case for successful completion of the generalization test in Phase 5. Exemplar training did appear to facilitate successful test performances on novel stimuli, particularly for Participant 15.

Participants 16, 17, and 18. Early on, Participants 16, 17, and 18 presented difficulties in completing the initial phases that appeared to be more serious than the lapses demonstrated by the previous group of children (see Table 6). Specifically, Participants 16 and 18 both failed on their first exposure to Phase 1 , and all three children showed considerable difficulty in completing the contextual control training in Phase 2. Therefore, a second intervention involving familiar stimulus sets of word pairings was employed (see O'Connor, Rafferty, Barnes-Holmes, \& BarnesHolmes, 2009).

For illustrative purposes, consider the performances of Participant 16. He continually failed to reach criterion in Phase 2, in spite of four exposures. At this point, a set of familiar written and spoken word pairs (Sets 7 and 8) replaced the abstract stimuli. Although his Phase 2 performance did not improve on the first exposure, he reached criterion on the second and moved swiftly through Phases 3 and 4 . However, he produced a very weak performance on the generalization test and was immediately exposed to exemplar training with the same familiar stimulus set. He completed this training in the minimum number of trials and was thus reexposed to all five phases on novel but familiar sets (Sets 9 and 10). When he passed the test immediately, he was exposed to the training and testing using Sets 1 and 2, which included nonsense syllables and abstract shapes, and passed immediately. A very similar pattern overall was recorded for Participants 17 and 18, both of whom required training with familiar stimuli and exemplar training. Again, both interventions appeared equally effective.

Taken together, Experiments 1 and 2 demonstrate a range of competencies in terms of the children's abilities to demonstrate and learn 
Table 6

The Training and Test Performances Recorded With Participants 16-18 Across All Phases in Experiment 2

\begin{tabular}{|c|c|c|c|c|c|c|c|}
\hline Participant & Sets & $\begin{array}{c}\text { Phase } 1 \\
\text { training (20) }\end{array}$ & $\begin{array}{c}\text { Phase } 2 \\
\text { training (40) }\end{array}$ & $\begin{array}{c}\text { Phase } 3 \\
\text { testing (20) }\end{array}$ & $\begin{array}{c}\text { Phase } 4 \\
\text { training (20) }\end{array}$ & $\begin{array}{l}\text { Phase } 5 \\
\text { testing (20) }\end{array}$ & $\begin{array}{c}\text { Phase } 6 \\
\text { training (20) }\end{array}$ \\
\hline \multirow{9}{*}{16} & \multirow{5}{*}{$1 \& 2$} & 15 & - & - & - & - & - \\
\hline & & 20 & 29 & - & - & - & - \\
\hline & & 20 & 25 & - & - & - & - \\
\hline & & 20 & 28 & - & - & - & - \\
\hline & & 20 & 29 & - & - & - & - \\
\hline & \multirow{2}{*}{$7 \& 8$} & 20 & 25 & - & - & - & - \\
\hline & & 20 & 39 & 20 & 20 & 10 & 18 \\
\hline & $9 \& 10$ & 20 & 39 & 20 & 20 & 19 & - \\
\hline & $1 \& 2$ & 20 & 39 & 20 & 20 & 20 & - \\
\hline \multirow{10}{*}{17} & \multirow{6}{*}{$1 \& 2$} & 19 & 23 & - & - & - & - \\
\hline & & 20 & 25 & - & - & - & - \\
\hline & & 20 & 31 & - & - & - & - \\
\hline & & 18 & 26 & - & - & - & - \\
\hline & & 18 & 21 & - & - & - & - \\
\hline & & 20 & 25 & - & - & - & - \\
\hline & $7 \& 8$ & 20 & 39 & 20 & 20 & 16 & 20 \\
\hline & \multirow{2}{*}{$9 \& 10$} & 20 & 39 & 20 & 20 & 2 & 20 \\
\hline & & 20 & 38 & 20 & 20 & 19 & - \\
\hline & $1 \& 2$ & 20 & 39 & 18 & 20 & 19 & - \\
\hline \multirow{13}{*}{18} & \multirow{6}{*}{$1 \& 2$} & 16 & - & - & - & - & - \\
\hline & & 19 & 27 & - & - & - & - \\
\hline & & 19 & 25 & - & - & - & - \\
\hline & & 19 & 24 & - & - & - & - \\
\hline & & 20 & 31 & - & - & - & - \\
\hline & & 19 & 26 & - & - & - & - \\
\hline & \multirow{6}{*}{$7 \& 8$} & 20 & 30 & - & - & - & - \\
\hline & & 20 & 26 & - & - & - & - \\
\hline & & 20 & 25 & - & - & - & - \\
\hline & & 20 & 33 & - & - & - & - \\
\hline & & 20 & 39 & 17 & 20 & 8 & 17 \\
\hline & & 20 & 40 & 19 & 20 & 20 & - \\
\hline & $1 \& 2$ & 19 & 40 & 20 & 18 & 19 & - \\
\hline
\end{tabular}

Note. Dashed lines (-) indicate absence of exposure to a particular stimulus set. Each line represents an individual experimental session. 
contextually controlled symmetry and asymmetry. More important, although some of the children with autism presented with considerable difficulty in the target training and test phases, these weaknesses were easily rectified with interventions that included exemplar training and the use of familiar stimuli.

Because of this relative ease of remediation, we then sought an additional group of children that might also require remediation for the establishment of contextually controlled symmetry and asymmetry, with a view to examining alternative interventions that might be useful for such a population. This was the focus of Experiment 3.

\section{Experiment 3}

\section{Participants}

Five children independently diagnosed with mild to moderate autism participated in Experiment 3. All were boys between 5 and 8 years old (i.e., somewhat younger than the previous group). They were referred to as Participants 19 through 23. All five children attended CABAS facilities in Dublin, Drogheda, or Cork in Ireland. The children were classified in the CABAS system according to their individual levels of verbal behavior and were all categorized as reader/writers with emergent levels of self-editing skills. Thus, they had relatively high levels of verbal behavior with emerging reading and writing abilities.

\section{Setting}

All aspects of the setting were identical to the previous experiments.

\section{Materials}

The current experiment involved the use of Sets 1-4 as before (i.e., two with nonsense syllables and two with arbitrary shapes). Two additional sets (Sets 11 and 12) were also employed, and these differed considerably from the previous experiments. Specifically, Set 11 comprised an array of 10 familiar and nameable pictures (i.e., car, ball, spoon, block, hairbrush, pencil, chicken, tractor, shoe, and cup), each printed on a white card. Set 12 comprised two identical copies of 100 common pictures (e.g., two horses, two suns, etc.), each of which was also printed on white card. Some of these pictures would likely have been familiar and nameable by the participants. The same two contextual cues (a blue and a red circle) were employed. Once again, edible items and tokens were used as reinforcers for correct responding.

\section{Experimental Sequence}

The experimental sequence employed in Experiment 3 was similar to but more extensive than that employed previously. Specifically, Phases 1 through 5 were identical to the first two experiments, and four additional levels of intervention were inserted between the training of contextual control over symmetry and asymmetry in Phase 2 and the test in Phase 3 (see Table 7). 
Table 7

The Experimental Sequence Conducted in Experiment 3

\begin{tabular}{|c|c|c|}
\hline Phase 1 & Explicit name training (spoken-written) & $\begin{array}{l}\text { Set } 1 \\
\text { A1-B1 \& A2-B2 }\end{array}$ \\
\hline Phase 2 & Train symmetry (C1) and asymmetry (C2) under contextual control & $\begin{array}{l}\text { Set } 1 \\
\text { C1 (B1-A1 \& B2-A2) } \\
\text { C2 (B1-A2 \& B2-A1) }\end{array}$ \\
\hline $\begin{array}{l}\text { Intervention } \\
\text { Level } 1\end{array}$ & $\begin{array}{l}\text { What is it? Give me the right answer. } \\
\text { What is it? Give me the wrong answer. }\end{array}$ & Set 11 \\
\hline \multicolumn{3}{|c|}{ Phase 1-2 (as above) } \\
\hline $\begin{array}{l}\text { Intervention } \\
\text { Level } 2\end{array}$ & $\begin{array}{l}\text { Give me something. Give me a } \\
\text { Give me something. Don't give me a }\end{array}$ & Set 11 \\
\hline \multicolumn{3}{|c|}{ Phase 1-2 (as above) } \\
\hline $\begin{array}{l}\text { Intervention } \\
\text { Level } 3\end{array}$ & $\begin{array}{l}\text { Presence of contextual cues } \\
\text { Give me something. Give me a } \\
\text { Give me something. Don't give me a }\end{array}$ & Set 11 \\
\hline \multicolumn{3}{|c|}{ Phase 1-2 (as above) } \\
\hline $\begin{array}{l}\text { Intervention } \\
\text { Level } 4\end{array}$ & $\begin{array}{l}\text { Presence of contextual cues } \\
\text { Identity and nonidentity } \\
\text { Matching in an MTS format }\end{array}$ & Set 12 \\
\hline \multicolumn{3}{|c|}{ Phase 1-2 (as above) } \\
\hline Phase 3 & $\begin{array}{l}\text { Test symmetry }(\mathrm{C} 1) \text { and } \\
\text { asymmetry }(\mathrm{C} 2) \text { under contextual control }\end{array}$ & $\begin{array}{l}\text { Set } 1 \\
\text { C1 (B1-A1 \& B2-A2) } \\
\text { C2 (B1-A2 \& B2-A1) }\end{array}$ \\
\hline Phase 4 & Conditional discrimination training in an MTS format & $\begin{array}{l}\text { Set } 2 \\
\text { A3-B3 \& A4-B4 }\end{array}$ \\
\hline Phase 5 & $\begin{array}{l}\text { Test generalized symmetry }(\mathrm{C} 1) \text { and } \\
\text { asymmetry }(\mathrm{C} 2) \text { under contextual control in an MTS format }\end{array}$ & $\begin{array}{l}\text { Set } 2 \\
\text { C1 (B3-A3 \& B4-A4) } \\
\text { C2 (B3-A4 \& B4-A3) }\end{array}$ \\
\hline
\end{tabular}

The additional intervention levels were introduced for participants who failed to reach criterion in Phase 2. The four levels of the intervention were introduced sequentially, and each was separated by reexposure to the original phases. That is, participants who failed to reach criterion in Phase 2 were immediately exposed to Intervention Level 1. Once they reached criterion here, they were reexposed to Phases 1 and 2. Participants who passed Phase 2 at this point proceeded immediately to Phase 3, as in the previous experiments. Participants who failed were then exposed to Intervention Level 2. This pattern continued until participants had passed all five phases of the original procedure.

\section{Corrective Feedback}

All aspects of corrective feedback were identical to Experiments 1 and 2.

\section{Procedure}

The amount of training and testing to which each child was exposed depended on his or her performance during each phase and/or the level of the intervention. Each child was exposed to one experimental session three to four days per week (availability permitting). Each session lasted approximately $20 \mathrm{~min}$, with a break of several minutes at an appropriate point 
approximately halfway through the session. If, at any point during training or testing, a child asked to stop or indicated the desire to stop, the session was terminated for that day and the trials in question were repeated in the following session.

Intervention Level 1. Intervention at Level 1 was primarily concerned with training the children to appropriately emit either the correct or incorrect naming response during a task, as a means of establishing a pattern of responding that would facilitate appropriate asymmetry responding in Phase 2. All training trials in Level 1 involved the presentation of a random sample of five of the pictures from Set 11. These were placed down on the table in front of the participant. On each trial, the child was provided with one of two antecedents that required the right or wrong answer. For example, if the five pictures placed on the experimental table depicted a ball, a horse, a spoon, a shoe, and a cup, the experimenter pointed to one of the pictures (e.g., the spoon) and the child was asked, "What is it? Give me the right answer" or "What is it? Give me the wrong answer." A correct response was only recorded if the child emitted the appropriate name (this was either correct or incorrect depending upon the experimenter's instruction). An incorrect response was recorded if the child emitted an inappropriate name or failed to make a response within $10 \mathrm{~s}$ of the instruction. After each incorrect response, the experimenter emitted the appropriate name and the next trial was presented immediately. After the provision of an appropriate response (either by the participant or the experimenter), the five pictures were removed from the table and the next trial involving an alternative sample of five pictures was presented immediately.

Level 1 comprised blocks of 20 randomized trials, requesting 10 correct naming responses and 10 incorrect naming responses. A random array of five pictures from Set 11 was presented on every trial. Participants were required to reach a criterion of 20 consecutively correct responses, and training continued until this occurred.

Intervention Level 2. Level 2 was similar in format to Level 1 but attempted to train the children to make an appropriate selection response that was either correct or incorrect from an array of five pictures. On each trial, they were instructed, for example, "Give me something. Give me a chicken" or "Give me something. Don't give me a chicken." A correct response consisted of the participant selecting an appropriate picture based on the experimenter's instruction. Incorrect responses were consequated with corrective feedback, and the experimenter then modeled the correct response by selecting an appropriate item. Once again, 10 trials were designated for selecting the correct picture and 10 trials for selecting an incorrect picture.

Intervention Level 3. Level 3 was similar in format to Level 2 but reintroduced the contextual cues in order to establish contextually controlled correct and incorrect responding. During each trial, the experimenter placed one of the cues on the table above the middle picture. One cue was designated as controlling correct responding, with the other controlling incorrect responding. The instruction provided by the experimenter depended upon the cue presented. That is, in the presence of the blue circle, the participant was instructed, for example, "Give me something. Give me a horse." Alternatively, in the presence of the red circle, the participant was instructed, for example, "Give me something. Don't give me a ball." 
Intervention Level 4 . Level 4 differed in format to the three previous levels of the intervention because trials were presented in the form of an identity matching task using an MTS format (in order to resemble Phase 4). All trials involved stimulus pairs from Set 12. During each trial, the experimenter placed two identical pictures and a third randomly selected picture on the table. One card from the matching pair was presented as a sample stimulus, with the two remaining cards presented as comparisons. One of the contextual cues was then placed above the sample. During these trials, no verbal antecedent was provided, and participants were required to make a selection response that was appropriate in the presence of the contextual cue. That is, in the presence of one cue, participants were required to point to or touch the comparison that matched the sample. Alternatively, in the presence of the other cue, participants were required to select the comparison that did not match the sample. A response was recorded as correct when participants selected the appropriate comparison in the presence of a specific contextual cue. All other aspects of the format of Level 4 were identical to the previous level.

\section{Interobserver Agreement}

Twenty-five percent of all test trials and 30\% of all training trials were recorded by an independent observer who was located at the opposite end of the experimental table from the participant and experimenter. This observer did not interact with the children in any way during the sessions. There was $100 \%$ agreement on all trials observed by both the experimenter and the independent observer.

\section{Results}

The total numbers of training trials required to reach criterion and the total numbers of correct test trials recorded for all participants across all five phases and the four levels of the intervention (as appropriate) in Experiment 3 are presented in Table 8. Each participant's results are summarized individually below.

Participant 19. Participant 19 produced a weak performance during initial training of contextually controlled symmetry and asymmetry (24/40) but reached criterion in Level 1 of the intervention in almost the minimum number of trials (22). He thereafter completed all five phases with ease.

Participant 20. Participant 20 showed some initial difficulty in completing the explicit name training in Phase 1 and only did so after three exposures. When he also failed to reach criterion during his first exposure to Phase 2 (30/40), he proceeded to Level 1 of the intervention. Although he completed this in almost the minimum number of training trials, he still failed to reach criterion in a second exposure to Phase 2 (34/40). He completed Level 2 of the intervention in the minimum number of trials and subsequently completed Phase 2 in his third exposure (37/40). He completed Phases 3 through 5 immediately thereafter.

Participant 21. Participant 21 easily proceeded through the name training but produced weak performances on his first exposure to Phase 2 $(22 / 40)$. He required 31 training trials to complete Level 1 of the intervention, but this appeared to have no impact on Phase 2 performances, when 
he produced only 23/40 on his second exposure. He completed Level 2 of the intervention in the minimum number of trials. Although this appeared to have a more positive impact on Phase 2 performances, with an improvement to $32 / 40$, it still constituted a fail. Hence, he was immediately exposed to Level 3 of the intervention. He completed this within only 25 trials and subsequently completed Phase 2 on the fourth exposure. He completed Phases 3 through 5 immediately thereafter.

Table 8

The Training and Test Performances Recorded With Participants 19-23 Across All Phases in Experiment 3

\begin{tabular}{|c|c|c|c|c|c|c|c|c|c|}
\hline \multirow[b]{2}{*}{ Part. } & \multirow{2}{*}{$\begin{array}{l}\text { Phase } 1 \\
\text { training } \\
(20)\end{array}$} & \multirow{2}{*}{$\begin{array}{c}\text { Phase } 2 \\
\text { training } \\
(40)\end{array}$} & \multicolumn{4}{|c|}{ Intervention levels } & \multirow{2}{*}{$\begin{array}{c}\text { Phase } 3 \\
\text { testing } \\
(20)\end{array}$} & \multirow{2}{*}{$\begin{array}{c}\text { Phase } 4 \\
\text { training } \\
(20)\end{array}$} & \multirow{2}{*}{$\begin{array}{l}\text { Phase } 5 \\
\text { training } \\
\text { (20) }\end{array}$} \\
\hline & & & Level 1 & Level 2 & Level 3 & Level 4 & & & \\
\hline \multirow{2}{*}{19} & 19 & 24 & 22 & - & - & - & - & - & - \\
\hline & 20 & 38 & - & - & - & - & 20 & 18 & 18 \\
\hline \multirow{5}{*}{20} & 14 & - & - & - & - & - & - & - & - \\
\hline & 11 & - & - & - & - & - & - & - & - \\
\hline & 20 & 30 & 21 & - & - & - & - & - & - \\
\hline & 19 & 34 & - & 20 & - & - & - & - & - \\
\hline & 18 & 37 & - & - & - & - & 19 & 19 & 18 \\
\hline \multirow{4}{*}{21} & 18 & 22 & 31 & - & - & - & - & - & - \\
\hline & 19 & 23 & - & 20 & - & - & - & - & - \\
\hline & 20 & 32 & - & - & 25 & - & - & - & - \\
\hline & 20 & 37 & - & - & - & - & 18 & 17 & 17 \\
\hline \multirow{5}{*}{22} & 16 & 21 & 89 & - & - & - & - & - & - \\
\hline & 20 & 24 & - & 28 & - & - & - & - & - \\
\hline & 20 & 33 & - & - & 20 & - & - & - & - \\
\hline & 20 & 37 & - & - & - & - & 17 & 11 & - \\
\hline & - & - & - & - & - & - & - & 20 & 16 \\
\hline \multirow{5}{*}{23} & 19 & 26 & 28 & - & - & - & - & - & - \\
\hline & 20 & 27 & - & 27 & - & - & - & - & - \\
\hline & 20 & 28 & - & - & 20 & - & - & - & - \\
\hline & 18 & 26 & - & - & - & 61 & - & - & - \\
\hline & 19 & 40 & - & - & - & - & 19 & 18 & 19 \\
\hline
\end{tabular}

Note. Dashed lines (-) indicate absence of exposure to a particular stimulus set. Each line represents an individual experimental session.

Participant 22. Participant 22 also required three levels of training in the intervention. However, he differed primarily from Participant 21 in that he required 89 training trials to reach criterion at Level 1. He required both 
Levels 2 and 3 of the intervention in order to pass Phase 2 but then failed to reach criterion in the MTS training in Phase 4 . We decided at this point to let the child proceed to Phase 4 testing, during which he produced a perfect performance. He then completed Phase 5 immediately.

Participant 23. Participant 23 was the only child who required exposure to all four levels of the intervention. Although he completed the first three levels in relatively small numbers of trials, he continued to fail to reach criterion when transferred back to Phase 2. It took no less than 61 trials for him to complete Level 4, but this appeared to have a significant impact on Phase 2 performances. He completed Phases 3 through 5 immediately thereafter.

\section{General Discussion}

The data obtained across Experiments 1-3 indicated a range of competencies in the children's abilities to learn and generalize contextually controlled symmetry and asymmetry performances. Specifically, the eight typically developing children (Experiment 1) and four of the children with autism (Experiment 2, Participants 9-12) proceeded rapidly through all phases and readily demonstrated the target generalized performances, without specific intervention. Three of the remaining children with autism from Experiment 2 (Participants 13-15) learned the contextually controlled symmetry and asymmetry with little difficulty but failed to demonstrate generalized performances in the final test. These difficulties were readily overcome with exemplar training of the target relations, followed by successful generalization to a novel set of abstract stimuli. In contrast, the final three children with autism from Experiment 2 (Participants 16-18) showed difficulties earlier in their exposure to the symmetry and asymmetry trials and failed to reach criterion on these tasks when abstract stimuli were employed. Switching to familiar stimulus sets in training successfully established the target contextual control in the first instance. However, this group of children also required multiple-exemplar training in order to show the generalization of the target responses with abstract stimuli.

The findings from Experiment 3 indicated the benefits of an additional intervention for those children who showed particular difficulty in acquiring the target performances. Specifically, one child (Participant 19) required one level of training; one (Participant 20) required two levels; two (Participants 21 and 22) required three levels; and one (Participant 23) required all four levels to establish the target repertoires. The four-level intervention was specifically concerned with explicit training of correct and incorrect responding across various response forms. The positive outcomes added to those recorded in the Experiment 2.

Several studies have attempted to demonstrate the relationships among, and possible substitutability of, members of an equivalence class. In short, the existing data demonstrate that relations among stimuli once formed may change individually, or they may change en masse, depending on various experimental and preexperimental conditions (Pilgrim \& Galizio, 1995; Spradlin, Cotter, \& Baxley, 1973). In an attempt to account for the variability in experimental outcomes, especially after reversal procedures, Carrigan and Sidman (1992) postulated the distinction between Type S and Type R responding, which distinguished between control by the positive or the negative comparison stimulus. According to these authors, reversing baseline 
conditional discriminations reverses symmetry (but not equivalence) by encouraging a switch from Type $S$ to Type R control.

The current study focused specifically on the distinction between symmetry and asymmetry and employed an intervention based almost entirely on training the children to switch between correct and incorrect responding (i.e., between the positive comparison and a negative comparison). One might conceptualize the training, therefore, as the provision of contingencies for explicitly switching between Type S and Type R control, where S controlled symmetry responding and R controlled asymmetry responding. Without further research, however, it is not possible to draw this conclusion. Given the two-comparison format that was employed throughout the current study, it is not possible to know if a participant's matching response involved selecting or rejecting the correct stimulus on any given trial. Thus, the symmetry and asymmetry training may have established two separate patterns of Type S control, two patterns of Type R control, or some combination thereof within and across participants. At the current time, therefore, it seems wise simply to refer to the performances as symmetry and asymmetry and avoid inferring particular types of stimulus control that extend beyond the available data.

The current findings indicated that all of the typically developing children and some of those with autism learned to switch between symmetry and asymmetry with ease, whereas other children with autism needed specific interventions to acquire these skills. For those who demonstrated the greatest deficits in this regard, an intervention that comprised a breakdown of the component skills in conjunction with contingencies for switching between correct and incorrect responding proved to be highly effective, at one level or another. These outcomes appear to support the view that the children were in fact being trained to switch explicitly between symmetry and asymmetry. The current study offers the first empirical evidence of these effects with children.

The present findings support the RFT view of the importance of flexibility in relational responding. From this perspective, the abilities of relating one stimulus to another, and of combining the relations among stimuli, are flexible behavioral repertoires under specific environmental control. Indeed, one might conceptualize the current intervention in Experiment 3 as explicit training in flexibility. Relational frame theory emphasizes the importance of flexibility in the underlying behavioral processes, rather than being concerned with flexibility in the content itself (see Healy, Barnes-Holmes, \& Smeets, 2000). Several authors have recently argued for the need to facilitate flexibility in programs designed to establish relational repertoires, especially in populations for whom cognitive rigidity characteristically interferes with learning (e.g., autism spectrum disorder; O’Hora, Pelaez, \& BarnesHolmes, 2005; O’Toole \& Barnes-Holmes, 2009). Indeed, children must learn to switch relational responding between correct and incorrect, more and less, up and down, over and under, similar and opposite, and so on as part of many aspects of standard educational curricula. The positive outcomes associated with the current intervention in Experiment 3 point to the importance of flexibility as a key feature of relational repertoires.

It is perhaps interesting that deficits in this regard emerged with a group of children who already had considerable experience in educational facilities that offered individualized behavioral programs. However, the children in this case were being asked to produce the "wrong" answer, which is 
counterintuitive in the structured educational environments in which they are placed. As a result, one might assume that flexibility is neither an implicit result of these training regimes nor trained directly. Put another way, highly structured educational programs for populations prone to behavioral rigidity may exacerbate rather than alleviate rigidity. While it is likely the case that flexibility emerges across time with adequate training (the data obtained with the typically developing children in Experiment 1 support this view), the data also suggest that it may be important to explicitly establish flexibility as early as possible.

The current research arose from conflicting findings within the literature regarding the extent to which symmetry can be defined as operant behavior (Barnes-Holmes et al., 2001a, 2001b; Boelens et al., 2003). The results from Experiments 1 through 3 produced consistent evidence of generalized contextually controlled symmetry and asymmetry that clearly support the interpretation of symmetry and asymmetry as operant behavior. Furthermore, these skills were successfully established in Experiment 2 with the use of multiple-exemplar training, which has been reported to be successful in the establishment of generalized operant responding. Although these findings support those reported previously by Barnes-Holmes et al., they fail to support Boelens et al. However, the latter study did not include any attempt to demonstrate contextual control over the participants' performances, whereas the current study did. Hence, it is difficult to draw clear comparisons between the studies.

One of the most striking features of the data reported in the current study was the positive impact of the interventions employed. Across Experiments 2 and 3 in particular, the children were exposed to exemplar training, the use of familiar stimuli, or explicit training in correct and incorrect responding as a means of establishing the target symmetry and asymmetry performances in Phase 2. Because no systematic comparison of these interventions was conducted, it is difficult to compare their relative effects in a precise manner. However, when familiar stimuli were employed in Experiment 2, the children emitted the appropriate response patterns in Phase 2 but failed to generalize these performances later in Phase 5 . In contrast, when the intervention was employed at the same point in Experiment 3 , the children also emitted the appropriate response patterns in Phase 2 and then immediately demonstrated the generalization of these performances in Phase 5, without requiring additional intervention. As a result, the latter intervention may be perceived to be more effective. In any case, it is important to have at hand a range of specific interventions, some of which may better suit the needs of one child over another, and the current experiments showed evidence of considerable variability within and across the target groups of children who participated.

\section{References}

BARNES-HOLMES, Y., BARNES-HOLMES, D., ROCHE, B., \& SMEETS, P. M. (2001a). Exemplar training and a derived transformation of function in accordance with symmetry. The Psychological Record, 51, 287-308.

BARNES-HOLMES, Y., BARNES-HOLMES, D., ROCHE, B., \& SMEETS, P. M. (2001b). Exemplar training and a derived transformation of function in accordance with symmetry: II. The Psychological Record, 51, 589-604. 
BOELENS, H., VAN DEN BROEK, M., \& CALMEYN, S. (2003). Is children's symmetry matching-to-sample the product of symmetric experiences with spoken names? The Psychological Record, 53, 593-615.

CARR, D., \& BLACKMAN, D. E. (2001). Relations among equivalence, naming and conflicting baseline control. Journal of the Experimental Analysis of Behavior, 75, 55-76.

CARRIGAN, P. F., \& SIDMAN, M. (1992). Conditional discrimination and equivalence relations: A theoretical analysis of control by negative stimuli. Journal of the Experimental Analysis of Behavior, 58, 183-204.

DOUGHER, M., PERKINS, D. R., GREENWAY, D., KOONS, A., \& CHIASSON, C. (2002). Contextual control of equivalence based transformation of functions. Journal of the Experimental Analysis of Behavior, 78, 63-93.

GREER, R. D., \& ROSS, D. E. (2007). Verbal behavior analysis: Inducing and expanding new verbal capabilities in children with language delays. Boston: Pearson Press.

HEALY, O., BARNES-HOLMES, D., \& SMEETS, P. M. (2000). Derived relational responding as generalized operant behavior. Journal of the Experimental Analysis of Behavior, 74, 207-227.

JOHNSON, C., \& SIDMAN, M. (1993). Conditional discrimination and equivalence relations: Control by negative stimuli. Journal of the Experimental Analysis of Behavior, 59, 333-347.

MEEHAN, E. L., \& FIELDS, L. (1995). Contextual control of new equivalence classes. The Psychological Record, 45, 165-182.

MICHAEL, R. L., \& BERNSTEIN, D. J. (1991). Transient effects of acquisition history on generalization in a matching-to-sample task. Journal of the Experimental Analysis of Behavior, 56, 155-166.

O'CONNOR, J., RAFFERTY, A., BARNES-HOLMES, D., \& BARNES-HOLMES, Y. (2009). The role of verbal behavior, stimulus nameability, and familiarity on the equivalence performances of autistic and normally developing children. The Psychological Record, 59, 53-74.

O'HORA, D., PELAEZ, M., \& BARNES-HOLMES, D. (2005). Derived relational responding and performance on verbal subtests of the WAIS-III. The Psychological Record, 55, 155-175.

O'TOOLE, C., \& BARNES-HOLMES, D. (2009). Three chronometric indices of relational responding as predictors of performance on a brief intelligence test: The importance of relational flexibility. The Psychological Record, 59, 119-132.

PILGRIM, C., CHAMBERS, L., \& GALIZIO, M. (1995). Reversal of baseline relations and stimulus equivalence: II. Children. Journal of the Experimental Analysis of Behavior, 63, 239-254.

PILGRIM, C., \& GALIZIO, M. (1990). Relations between baseline contingencies and equivalence probe performances. Journal of the Experimental Analysis of Behavior, 54, 213-224.

PILGRIM, C., \& GALIZIO, M. (1995). Reversal of baseline relations and stimulus equivalence: I. Adults. Journal of the Experimental Analysis of Behavior, 63, 225-238.

SAUNDERS, R. R., DRAKE, K. M., \& SPRADLIN, J. E. (1999). Equivalence class establishment, expansion, and modification in preschool children. Journal of Experimental Analysis of Behavior, 71, 195-214. 
SAUNDERS, R. R., SAUNDERS, K. J., KIRBY, K. C., \& SPRADLIN, J. E. (1988). The merger and development of equivalence classes by unreinforced conditional selection of comparison stimuli. Journal of the Experimental Analysis of Behavior, 50, 145-162.

SIDMAN, M. (1986). Functional analysis of emergent verbal classes. In T. Thompson and M. D. Zeiler (Eds.), Analysis and integration of behavioral units (pp. 213-245). Hillsdale, NJ: Erlbaum.

SPRADLIN, J. E., COTTER, V. W., \& BAXLEY, N. (1973). Establishing a conditional discrimination without direct training: A study of transfer with retarded adolescents. American Journal of Mental Deficiency, 77, 556-566.

SPRADLIN, J. E., SAUNDERS, K. J., \& SAUNDERS, R. R. (1992). The stability of equivalence classes. In S. C. Hayes and L. J. Hayes (Eds.), Understanding verbal relations (pp. 29-42). Reno, NV: Context Press.

STEWART, I., BARNES-HOLMES, D., ROCHE, B., \& SMEETS, P. M. (2001). Generating derived relational networks via the abstraction of common physical properties: A possible model of analogical reasoning. The Psychological Record, 51, 381-408.

WULFERT, E., \& HAYES, S. C. (1988). The transfer of conditional sequencing through conditional equivalence classes. Journal of the Experimental Analysis of Behavior, 50, 125-144. 
FIRST-TIME INDIVIDUAL AND STUDENT SUBSCRIBERS PLEASE DEDUCT $\$ 10.00$ FROM THE SUBSCRIPTION FEE

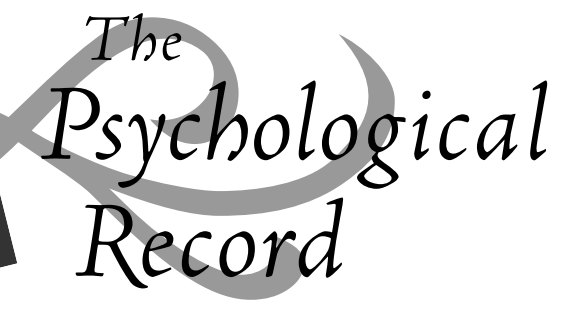

http://thepsychologicalrecord.siuc.edul

The Psychological Record is a quarterly journal of psychology. Since 1937, it has published psychological theory and research concerned with a broad range of topics in the discipline. Rapid publication of accepted manuscripts assures that each issue contains very recent work. The Psychological Record is edited by Ruth Anne Rehfeldt, PhD, BCBA-D, and published by the Rehabilitation Institute at Southern Illinois University.

\section{Selected Recent Articles}

Humans and Monkeys Exert Metacognitive Control Based on Learning Difficulty in a Perceptual Categorization Task. JJoshua S. Redford, Vol. 60, issue 4

Intertemporal Decision-Making for a Group. Richard Yi, Leila F. King, Anne E. Carter, Reid D. Landes, and Warren K. Bickel, Vol. 60, issue 4

Self-Control and Impulsiveness in Nondieting Adult Human Females: Effects of Visual Food Cues and Food Deprivation. Lori-Ann B. Forzano, John J. Chelonis, Caitlin Casey, Marion Forward, Jacqueline A. Stachowiak, and Jennifer Wood, Vol. 60 , issue 4

Characterizing Response-Reinforcer Relations in the Natural Environment: Exploratory Matching Analyses. Jolene R. Sy, John C. Borrero, and Carrie S. W. Borrero, Vol. 60, issue 4

A Comparison Among Worker Types Using a Composites Approach and Median Splits. Shahnaz Aziz, Karl L. Wuensch, and Howard R. Brandon, Vol. 60, issue 4

Proactive and Preventive Coping in Adjustment to College. Yiqun Gan, Yueqin $\mathrm{Hu}$, and Yiwen Zhang, Vol. 60, issue 4

Trained and Derived Relations With Pictures Versus Abstract Stimuli as Nodes. Erik Arntzen and Torunn Lian, Vol. 60, issue 4

The Implicit Relational Assessment Procedure as a Measure of Self-Esteem. C. Alix Timko, Erica L. England, James D. Herbert, and Evan M. Forman, Vol. 60, issue 4

Name (please print)

Address

City, State, Zip

Email

Mail to:

The Psychological Record

Rehabilitation Institute

Rehn Hall - Mail Code 4609

Southern Illinois University Carbondale

1025 Lincoln Drive

Carbondale IL 62901

\section{Subscription Prices}

USA International

\begin{tabular}{lllll} 
Students & $\$ 40$ & $\$ 50$ & - \\
Professional Individual & $\$ 50$ & $\$ 60$ & - \\
Library/Institution & $\$ 160$ & $\$ 170$ & - \\
\hline \hline
\end{tabular}

$2011 \square$ New $\square$ Renew

$\square$ Check (in US currency) made payable to

The Psychological Record

$\square$ Credit cards accepted: $\square$ Visa $\square$ MasterCard

Number

Security code 